\title{
Neurological abnormalities in recent-onset schizophrenia and Asperger-syndrome
}

\author{
Dusan Hirjak ${ }^{1}{ }^{*}$, Robert Christian Wolf ${ }^{1,2}$, Sabine C. Koch ${ }^{3}$, Laura Mehl ${ }^{1}$, Janna K. Kelbel ${ }^{1}$, \\ Katharina Maria Kubera ${ }^{1}$, Tanja Traeger ${ }^{4}$, Thomas Fuchs ${ }^{1}$ and Philipp Arthur Thomann ${ }^{1}$ \\ 1 Department of General Psychiatry, Center for Psychosocial Medicine, University of Heidelberg, Heidelberg, Germany \\ 2 Department of Psychiatry, Psychotherapy and Psychosomatics, Saarland University, Homburg, Germany \\ ${ }^{3}$ Department of Dance Movement Therapy, Faculty of Therapeutic Sciences, SRH University Heidelberg, Heidelberg, Germany \\ ${ }_{4}^{4}$ Personality, Psychological Assessment, and Psychological Methods, Department of Psychology, University of Koblenz Landau, Landau, Germany
}

\section{Edited by:}

Sebastian Walther, University Hospital of Psychiatry of Bern, Switzerland

Reviewed by:

Einar Patrick Wilder-Smith, National University of Singapore, Singapore

Tobias Bracht, Cardiff University Brain Research Imaging Centre, UK

Lise Docx, University of Antwerp,

Belgium

*Correspondence:

Dusan Hirjak, Department of General

Psychiatry, Center for Psychosocial

Medicine, University of Heidelberg

Voßstraße 4, D-69115 Heidelberg,

Germany

e-mail:dusan.hirjak@

med.uni-heidelberg.de
Background: Neurological abnormalities including a variety of subtle deficits such as discrete impairments in sensory integration, motor coordination (MOCO), and sequencing of complex motor acts are frequently found in patients with schizophrenia (SZ) and commonly referred to as neurological soft signs (NSS). Asperger-syndrome (AS) is characterized by sensory-motor difficulties as well. However, the question whether the two disorders share a common or a disease-specific pattern of NSS remains unresolved.

Method: A total of 78 age- and education-matched participants $[26$ patients with recentonset SZ, 26 individuals with AS, and 26 healthy controls $(\mathrm{HC})]$ were recruited for the study. Analyses of covariance (ANCOVAs), with age, years of education, and medication included as covariates, were used to examine group differences on total NSS and the five subscale scores. Discriminant analyses were employed to identify the NSS subscales that maximally discriminate between the three groups.

Results: Significant differences among the three groups were found in NSS total score and on the five NSS subscales. The clinical groups differed significantly in the NSS subscale MOCO. The correct discriminant rate between patients with SZ and individuals with AS was $61.5 \%$. The correct discriminant rate was $92.3 \%$ between individuals with AS and HC, and $80.8 \%$ between $\mathrm{SZ}$ patients and $\mathrm{HC}$, respectively.

Conclusion: Our findings provide new evidence for the presence of NSS in AS and lend further support to previously reported difficulties in movement control in this disorder. According to the present results, SZ and AS seem to be characterized by both quantitative and qualitative NSS expression.

Keywords: NSS, motor abnormalities, recent-onset schizophrenia, Asperger-syndrome

\section{INTRODUCTION}

Neurological soft signs (NSS) are neurological abnormalities including a variety of subtle deficits such as discrete impairments in sensory integration, motor coordination, sequencing of complex motor acts, clumsiness, and occurrence of primitive reflexes (1-3). A higher prevalence of NSS has been consistently demonstrated not only in patients with clinically manifest schizophrenia (SZ) but also in their non-psychotic first-degree relatives (4). Recent studies indicated that NSS are not only restricted to SZ but are also present in bipolar disorders, depression, obsessivecompulsive disorders (OCD), and other forms of psychosis (5). Nevertheless, previous studies have assessed the power of NSS to discriminate between $\mathrm{SZ}$ and other neuropsychiatric disorders. In particular, SZ patients have significantly higher NSS levels than individuals with $\operatorname{OCD}(6,7)$, alcohol dependence (8), bipolar disorders $(9,10)$, depression $(11)$, and mixed psychiatric diagnoses (12). From a neurobiological point of view, the prefix "soft" indicates that NSS refer to a non-specific or global cerebral dysfunction rather than to impairments of specific or distinct brain regions.
Recent magnetic resonance imaging (MRI) studies on SZ found that increased NSS levels are related to aberrant brain morphology within cortical (13-15) and subcortical regions (13, 16-20). Furthermore, the aforementioned studies converge on the conclusion that NSS should be discussed as potential endophenotypes for SZ (4).

In 1911, a renowned German psychiatrist named Eugen Bleuler introduced the concept of accessory and fundamental symptoms in SZ $(21,22)$. Accessory symptoms were non-specific state phenomena and comprised hallucinations, delusions, and catatonic signs (21). The fundamental symptoms were more specific to $\mathrm{SZ}$ and included autism, formal thought disorders, ambivalence, disorders of volition, affective-emotional, and affect-expressive changes $(21,22)$. Furthermore, when Bleuler (21) described the "autistic core" in SZ patients, he spoke about the withdrawal within the own inner world: "The most severe schizophrenics, who have no more contact with the outside world live in a world of their own. They have encased themselves with their desires and wishes [...]; they have cut themselves off as much as possible from any contact 
with the external world. This detachment from reality with the relative and absolute predominance of the inner life, we term autism" $[(21,23)$, p. 1122]. In general, the schizophrenic autism is characterized by a rich variety of clinical phenomena such as poor ability to interact with others, inaccessibility, negativistic tendencies, indifference, rigid attitudes, and behaviors, private hierarchy of values and goals, inappropriate expression and behavior, and idiosyncratic logic and thinking, respectively (22). Taken together, from the historical standpoint, SZ, and autism have been regarded as part of the same spectrum $(24,25)$.

In the early 40s, Kanner's (26) and Asperger's (27) use of the term autism changed it in the direction of its present meaning of disturbed social cognition. Subsequently, with the introduction of DSM-III in the late 1970s, autism became an independent diagnostic entity not being part of the diagnostic concept of SZ. However, problems with interpersonal contact, interaffective attunement, and perspective-taking, are core to both pathologies, though appearing in different forms, and hence, several studies provided empirical evidence for a diagnostic overlap in both disorders (28). Furthermore, clinical studies have shown that negative/deficit, disorganized, and motor symptoms are present in both individuals with autism and patients with SZ (29-31). More recently, before introduction of DSM-5, some authors even discussed an autism dimension for SZ (25). This suggests that distinguishing between both spectrum disorders remains a diagnostic challenge. Such diagnostic overlaps might confound the diagnosis and delay appropriate treatment of these patients. As a matter of fact, the symptoms overlap could at least partially account for the inconsistent findings in previous scientific studies.

Asperger-syndrome (AS) belongs to pervasive developmental disorders and is characterized by interaction and communication difficulties, and repetitive, stereotype, and restricted patterns of behavior. In fact, several clinical studies observed motor abnormalities in individuals with AS including involuntary dyskinesia, rigidity, hypotonia, abnormal posture and gait, clumsiness, reduced coordination of locomotor skills, and unstable balance, respectively (32-35). Some authors even consider motor abnormalities as a putative endophenotype for autism spectrum disorders (36). In the last decade, clinical research interest on motor abnormalities in autism has extended to the investigation of NSS in AS. However, there are only two studies which have investigated NSS prevalence in individuals diagnosed with AS $(33,34)$ and we are still lacking a profound understanding of NSS in AS.

Overall, the above mentioned clinical studies suggest that motor abnormalities are a typical characteristic of SZ and AS. Hence, there is a stimulating debate whether these disorders share similar sensory-motor features or not (37). Regarding subtle neurological deficits in autism, however, only Mayoral et al. (34) compared NSS in early-onset SZ and AS. Therefore, at present it is difficult to highlight a potential difference in subtle sensory-motor abnormalities in patients with SZ and individuals with AS. The precise evaluation of subtle sensory-motor neurological signs in SZ and AS is of potential clinical significance, since the assessment of NSS might allow for more accurate disease classification. Also, this approach might help to overcome the missing conceptual clarity and better delineate a precise phenotype in order to identify endophenotypes underpinning SZ and AS.
The purpose of this investigation was twofold. First, we were interested in whether there is a difference between NSS severity in patients with SZ and individuals with AS. Second, we sought to identify characteristic NSS, which are either unique or shared by both disorders. Based on the findings of a previous study in juveniles (34) and on our clinical observation, it was hypothesized that individuals with AS would show NSS scores at least as high as patients with SZ. Further, we expected AS individuals being predominantly susceptible to NSS that involve gross motor skills. Finally, we employed a descriptive and predictive linear discrimination analysis (LDA) in order to examine if both total NSS and subscale scores are able to discriminate between the three groups.

\section{MATERIALS AND METHODS \\ SUBJECTS}

The study sample consisted of 26 clinically stable patients with recent-onset SZ, 26 individuals with AS, and 26 healthy controls (HC) who participated in a larger study at the Department of General Psychiatry in Heidelberg, Germany as part of the Toward an Embodied Science of Intersubjectivity-Project (TESIS). The study sample was consecutively recruited between 2010 and 2013 from the Department of General Psychiatry in Heidelberg, Germany and from SALO GmbH in Ludwigshafen, Germany, a professional rehabilitation institution of education for autistic individuals. All participants were Caucasians. Study participants were excluded if: (1) they were aged $<18$ or $>35$ years, (2) they had a history of brain trauma or neurological disease, (3) they had a comorbid Axis-I- or -II-Disorder according to ICD-10 or DSM-IV, (4) they had shown alcohol/substance abuse or dependence within 24 months prior to participation, or (5) they had an IQ $<70$. Diagnoses of SZ and AS were made by specialized clinicians (DH and PAT) corresponding to DSM-IV criteria and supplemented by an extensive neuropsychological assessment. Clinical symptom determinations and structured clinical diagnostic interviews were conducted by trained clinical raters (Dusan Hirjak, Laura Mehl, and Janna K. Kelbel) and senior diagnosticians (Sabine C. Koch, Philipp Arthur Thomann). In particular, all study individuals were assessed for lifetime psychiatric diagnoses by trained psychiatrists (Dusan Hirjak and Philipp Arthur Thomann) via the German version of the Structured Clinical Interview for DSMIV (38) and reviews of hospital case notes. All participants in the AS group had previously received a clinical diagnosis of AS (F84.5) from an independent clinician according to standard criteria (a valid diagnosis of autism is an admission criterion for SALO GmbH). In addition, diagnoses of the participants with AS were confirmed with the Autism Diagnostic Observation Schedule [ADOS; (39)] administered by a trained and clinically experienced psychiatrist (Dusan Hirjak). In addition, IQ of individuals with AS (F84.5) has been systematically assessed with the German version of the Culture Fair Intelligence Test (CFT-20-R) (40). The intelligence in SZ patients and HC was not explicitly assessed, but clinically judged to be average or above average. Both patients with $\mathrm{SZ}$ and $\mathrm{HC}$ were required to have a leaving certificate from one of the secondary schools - Hautpschule (9 years), Realschule (10 years), or Gymnasium ( 13 years) - in order to participate in our study. The demographics and psychiatric history of the two clinical samples were retrieved from medical records. To examine the 
possible effect of medications on NSS, we standardized the dosage of antipsychotic medications chlorpromazine equivalents (CPZ). In healthy individuals, we used the PRIME early psychosis screening test [prevention through risk identification, management, and education (PRIME)] to screen for the presence of early psychotic symptoms, including information on any contact or treatment for any mental or psychological disorder (41). All study participants gave informed consent to participation, and the study has been approved by the local ethics committee of the Medical Faculty, University of Heidelberg, Germany.

\section{CLINICAL ASSESSMENTS}

Neurological soft signs were assessed using the Heidelberg Scale (2) that consists of five items assessing motor coordination (MOCO) (Ozeretski's test, diadochokinesia, pronation/supination, fingerto-thumb opposition, speech articulation), three items assessing integrative functions (IF) (station and gait, tandem walking, twopoint discrimination), two items assessing complex motor tasks (COMT) (finger-to-nose test, fist-edge-palm test), four items assessing right/left and spatial orientation (RLSO) (right/left orientation, graphesthesia, face-hand test, stereognosis), and two items assessing hard signs (HS) (arm holding test, mirror movements). Items were rated on a 0 (no prevalence) to 3 (marked prevalence) point scale. All items, with the exception of station and gait, tandem walking, right/left orientation, speech articulation, primitive reflexes, and Ozeretzki's test were rated separately on the right and left side. A sufficient internal reliability (Cronbach's alpha 0.83$)$ and high test-retest reliability $(0.88)$ have been established previously $(2,42)$. In the study conducted by Schröder and colleagues (2), to test the interrater reliability of the NSS scale, 42 patients and $\mathrm{HC}$ were simultaneously evaluated by two raters. The internal reliability of the scale was assessed by calculating Cronbach's $\alpha$. The testing procedure was generally standardized, but the explanations and the time required to complete the tasks were adjusted to the condition of the patients. In the present study, the NSS assessment has been conducted by two raters (Janna K. Kelbel and Laura Mehl) trained and supervised by the same psychiatrist (Dusan Hirjak). Both raters were blind to the main hypothesis of the study and investigated study participants independent of their diagnosis. Handedness was assessed on the Edinburgh Inventory (43). The severity of psychopathological symptoms was assessed with the Brief Psychiatric Rating Scale (BPRS) (44), the Scale for the Assessment of Positive Symptoms (SAPS) (45), and the Scale for the Assessment of Negative Symptoms (SANS) (46). Predictors of outcome were rated on the Strauss-Carpenter Scale (SCS) (47). The social, occupational, and psychological functioning in individuals with AS was assessed with the Global Assessment of Functioning (GAF) scale (48).

\section{CHARACTERISTICS OF PARTICIPANTS}

The three groups of participants were matched according to age and education. Level of IQ among individuals with AS ranged from 71 to 124 (mean IQ: $99.0 \pm 18.5$ ) according to CFT-20-R (40). Patients with SZ according to DSM-IV had an initial onset of psychosis within 2 years prior to study entry with a mean duration of illness of 7.15 months (range $2-15$ months). SZ subtypes were distributed as follows: paranoid $n=12$, disorganized $n=4$, and undifferentiated $n=10$. At the time of inclusion, all SZ patients were clinically stable with consistent medication doses for 4 weeks or longer. They were receiving treatment with a single second-generation antipsychotic agent according to their psychiatrists' choice. Patients were treated on average for $2.33 \pm 1.44$ months throughout the course of illness. Potential extrapyramidal side effects were excluded before study entry by an experienced psychiatrist who was not directly involved in the study. Individuals with AS and HC did not take any antipsychotic, mood stabilizing, anti-cholinergic, or antidepressive medications. SZ patients had low or no prevalence of positive and negative symptoms, as measured by SAPS (range: 0-74), SANS (range: 0-70), and BPRS. At the time of clinical and NSS assessment, no SZ patients manifested psychotic symptoms (two or more of the positive symptom items $>3$ or a total SAPS score $>40$ ).

\section{DATA ANALYSIS}

Data were analyzed using the Statistical Package of the Social Sciences (SPSS version 21.0, SPSS Inc., Chicago, IL, USA). Sociodemographic and clinical variables were described and compared between the three groups with unpaired $t$-test or chi-square test for categorical variables using conventional significance levels $(p<0.05)$. To test for differences in NSS performance between the three study subgroups, we conducted an analysis of covariance (ANCOVA) including the potentially distorting factors age, years of education, and CPZ. Gender comparisons on NSS performance within each study group and between the three groups used $t$-tests and analysis of covariance (ANCOVA). Further, $p$ values of the identified NSS subscales were corrected for the number of tested NSS subscales in our main analysis using the Bonferroni method. To this end, $\alpha$ was set to $p=0.05 / \mathrm{N}$, where $n(=18)$ equaled the number of correlations (classical Bonferroni correction). For this reason, the corrected threshold was set to $p=0.0027[\alpha=0.05 / 18$ tests (total NSS + five subscale scores $\times$ three groups)]. In a second step, a series of ANCOVAs considering age, years of education, and $\mathrm{CPZ}$ as covariates was conducted to further examine the differences between groups if a significant main effect was identified. Further, $p$ values of the identified NSS subscales were corrected for the number of tested NSS subscales using the Bonferroni method. To this end, $\alpha$ was set to $p=0.05 / \mathrm{N}$, where $n(=12)$ equaled the number of correlations (classical Bonferroni correction). For this reason, the corrected threshold was set to $p=0.0041[\alpha=0.05 / 12$ tests (total NSS + five subscale scores $\times$ two groups)]. Correlative analyses of SAPS, SANS, BPRS, and CPZ with total scores and subscores of NSS were conducted with the Pearson correlation coefficient.

To examine the ability of NSS to discriminate among the three groups, both descriptive and predictive LDAs were used (11, 49, 50 ). The aim of this analysis was to determine whether NSS subscales would discriminate between patients with SZ and those with AS. In this study, total NSS and the five subscale scores were treated as "within subject variable" (independent variables), whereas the diagnostic group was treated as the "between subject factor" (grouping variable). However, only those NSS scores that reached statistical significance in the ANCOVAs were used as predictive variables. 


\section{RESULTS}

Demographic characteristics of the patient groups and the $\mathrm{HC}$ are summarized in Table 1. Comparison of the three groups revealed a significant difference in gender (chi-square test: $\chi^{2}=8.35 ; \mathrm{df}=2$; $p=0.015)$ and $\operatorname{CPZ}[F(2,75)=85.16 ; p<0.001]$. There were no significant differences in age $[F(2,75)=0.55 ; p=0.577]$ and years of education $[F(2,75)=2.63 ; p=0.078]$ among the three groups. There was also no significant difference for BPRS scores between SZ patients and individuals with AS $[F(1,50)=2.9 ; p=0.094]$. There were no significant differences between male and female participants in NSS performance among the three groups. In addition, we found no significant differences between male and female individuals in the control group in any of NSS scores (Table 3). But, there was a significant gender difference in the performance on NSS subscale COMT in both individuals with AS and SZ patients (Table 3). However, this effect is most likely driven by the influence of confounders such as age, education, and medication, since a significant gender effect diminished after covarying for these factors.

\section{GROUP DIFFERENCE IN NSS SCORES (ANCOVA: CONTROLLING FOR AGE, YEARS OF EDUCATION, AND MEDICATION)}

Table 1 shows the prevalence of NSS across the three groups. Significant differences after controlling for age, years of education, and CPZ were found in NSS total score $[F(5,72)=14.7 ; p<0.001]$ and on the five NSS subscales MOCO $[F(5,72)=11.5 ; p<0.001]$, $\operatorname{IF}[F(5,72)=3.41 ; p=0.008], \operatorname{COMT}[F(5,72)=8.9 ; p<0.001]$, RLSO $[F(5,72)=4.02 ; p=0.003]$ and HS $[F(5,72)=3.2$; $p=0.012]$ among the three groups (Figure 1; Table 2). Further, $p$ values of the identified NSS subscales were corrected for the number of tested NSS subscales in our main analysis using the Bonferroni method $(p<0.0027)$. NSS total and two subscale scores (MOCO and COMT) hold Bonferroni correction for multiple testing.

\section{AS VS. SZ}

The ANCOVA showed that compared with SZ patients, the individuals with AS showed significantly higher NSS total scores $[F(4,47)=3.63 ; p=0.012]$ and higher scores on the subscale $\operatorname{COMT}[F(4,47)=4.2 ; p=0.005]$. However, individuals with AS showed lower scores on the NSS subscale MOCO $[F(4,47)=4.38$; $p=0.004]$ when compared to SZ patients. Further, $p$ values of the two identified NSS subscales were corrected for the number of tested NSS subscales in our main analysis using the Bonferroni method $(p<0.0041)$. Only the NSS subscale, MOCO hold Bonferroni correction for multiple testing. No significant difference was found between individuals with AS and SZ patients on the

Table 1 | Descriptive summary of the sociodemographic and clinical variables of all participants.

\begin{tabular}{|c|c|c|c|}
\hline Variable & Asperger-syndrome $(n=26)$ & Schizophrenia ( $n=26)$ & Healthy controls $(n=26)$ \\
\hline Mean age, years (SD) & $22.76 \pm 3.81$ & $23.38 \pm 3.87$ & $23.58 \pm 3.77$ \\
\hline \multicolumn{4}{|l|}{ Gender, $n$} \\
\hline Female & $8(30.8 \%)$ & $17(65.3 \%)$ & $17(65.3 \%)$ \\
\hline \multicolumn{4}{|l|}{ Handedness, $n$} \\
\hline Mean education, years (SD) & $12.03 \pm 1.84$ & $12.07 \pm 1.32$ & $12.8 \pm 0.63$ \\
\hline Mean duration of illness, months (SD) & - & $7.15 \pm .31$ & - \\
\hline Mean antipsychotic dose(CPZ) (SD) & 0 & $435.11 \pm 240.4$ & 0 \\
\hline Mean NSS score (SD) & $16.19 \pm 6.71($ median $=14.5)$ & $14.92 \pm 7.54($ median $=16.0)$ & $5.57 \pm 3.08($ median $=5.5)$ \\
\hline MOCO & $6.23 \pm 3.31$ & $6.79 \pm 3.91$ & $2.11 \pm 1.55$ \\
\hline Mean $\mathrm{ADOS}^{\mathrm{a}}$ (SD) & $13.61 \pm 3.27$ & - & - \\
\hline Mean SAPSb (SD) & - & $20.11 \pm 13.98$ & - \\
\hline Mean SANSc (SD) & $41.07 \pm 15.78$ & $30.69 \pm 18.81$ & - \\
\hline Mean BPRS ${ }^{d}$ (SD) & $29.34 \pm 15.49$ & $23.0 \pm 10.94$ & - \\
\hline Mean $\mathrm{SCS}^{\mathrm{e}}(\mathrm{SD})$ & - & $39.0 \pm 15.72$ & - \\
\hline Mean $\mathrm{GAF}^{f}(\mathrm{SD})$ & $63.96 \pm 10.67$ & - & - \\
\hline
\end{tabular}

Mean \pm standard deviation (SD).

${ }^{a}$ Autism Diagnostic Observation Schedule.

${ }^{b}$ Scale for the assessment of negative symptoms.

'Scale for the assessment of positive symptoms.

${ }^{a}$ Brief Psychiatric Rating Scale.

- Strauss-Carpenter Scale.

${ }^{f}$ Global Assessment of Functioning. 
Table 2 | Group differences in NSS performance.

\begin{tabular}{|c|c|c|c|c|c|c|c|c|}
\hline NSS measure & \multicolumn{2}{|c|}{ AS vs. SZ vs. HC } & \multicolumn{2}{|c|}{ AS vs. SZ } & \multicolumn{2}{|c|}{ AS vs. HC } & \multicolumn{2}{|c|}{ SZ vs. HC } \\
\hline MOCO & 11.5 & $<0.001$ & 4.38 & 0.004 & 15.29 & $<0.001$ & 11.33 & $<0.001$ \\
\hline COMT & 8.9 & $<0.001$ & 4.2 & 0.005 & 17.81 & $<0.001$ & 2.45 & 0.059 \\
\hline IF & 3.41 & 0.008 & 2.51 & 0.054 & 3.71 & 0.018 & 2.59 & 0.048 \\
\hline HS & 3.2 & 0.012 & 1.1 & 0.364 & 3.25 & 0.03 & 4.48 & 0.004 \\
\hline
\end{tabular}

ANCOVA = controlling for age, years of education and medication (CPZ); MOCO, motor coordination; COMT, complex motor tasks; IF, integrative function; RLSO, right/left and spatial orientation; HS, hard signs; AS, Asperger-syndrome; SZ, schizophrenia patients; HC, healthy controls. Differences surviving Bonferroni correction in bold.

Table 3 | Gender differences in NSS performance (two-tailed $t$-tests).

\begin{tabular}{|c|c|c|c|c|c|c|c|c|c|c|c|c|}
\hline \multirow[t]{2}{*}{ NSS measures } & \multicolumn{4}{|c|}{ Asperger-syndrome } & \multicolumn{4}{|c|}{ Schizophrenia } & \multicolumn{4}{|c|}{ Healthy controls } \\
\hline & $\begin{array}{l}\text { Male } \\
(n=18)\end{array}$ & $\begin{array}{l}\text { Female } \\
(n=8)\end{array}$ & $\begin{array}{l}t \\
(d f=24)\end{array}$ & $p$ & $\begin{array}{l}\text { Male } \\
(n=9)\end{array}$ & $\begin{array}{l}\text { Female } \\
(n=17)\end{array}$ & $\begin{array}{l}t \\
(\mathrm{df}=24)\end{array}$ & $p$ & $\begin{array}{l}\text { Male } \\
(n=9)\end{array}$ & $\begin{array}{l}\text { Female } \\
(n=17)\end{array}$ & $\begin{array}{l}t \\
(d f=24)\end{array}$ & $p$ \\
\hline NSS total score & $16.5 \pm 6.86$ & $15.5 \pm 6.76$ & -0.34 & 0.73 & $11.66 \pm 7.77$ & $16.64 \pm 7.04$ & 1.65 & 0.11 & $5.88 \pm 3.10$ & $5.41 \pm 3.16$ & -0.36 & 0.71 \\
\hline Moco & $6.55 \pm 3.72$ & $5.5 \pm 2.13$ & -0.74 & 0.46 & $5.44 \pm 4.15$ & $7.47 \pm 3.71$ & 1.27 & 0.21 & $2.11 \pm 1.69$ & $2.11 \pm 1.53$ & 0.01 & 0.99 \\
\hline COMT & $3.27 \pm 1.70$ & $1.87 \pm 1.14$ & -2.08 & 0.04 & $0.66 \pm 1.65$ & $2.11 \pm 1.49$ & 2.26 & 0.03 & $0.66 \pm 1.0$ & $0.58 \pm 087$ & -0.2 & 0.83 \\
\hline RLSO & $2.77 \pm 1.80$ & $3.75 \pm 3.49$ & 0.94 & 0.35 & $1.44 \pm 1.87$ & $1.76 \pm 1.95$ & 0.4 & 0.69 & $0.55 \pm 0.88$ & $0.94 \pm 1.14$ & 0.87 & 0.38 \\
\hline HS & $1.38 \pm 1.81$ & $1.50 \pm 1.30$ & 0.15 & 0.87 & $1.33 \pm 1.0$ & $1.94 \pm 1.47$ & 1.1 & 0.28 & $0.88 \pm 1.16$ & $0.47 \pm 0.71$ & -1.13 & 0.26 \\
\hline
\end{tabular}

Mean $\pm S D ; M O C O$, motor coordination; COMT, complex motor tasks; IF, integrative function; RLSO, right/left and spatial orientation; HS, hard. Bold font indicates significant differences $(p<0.05)$.

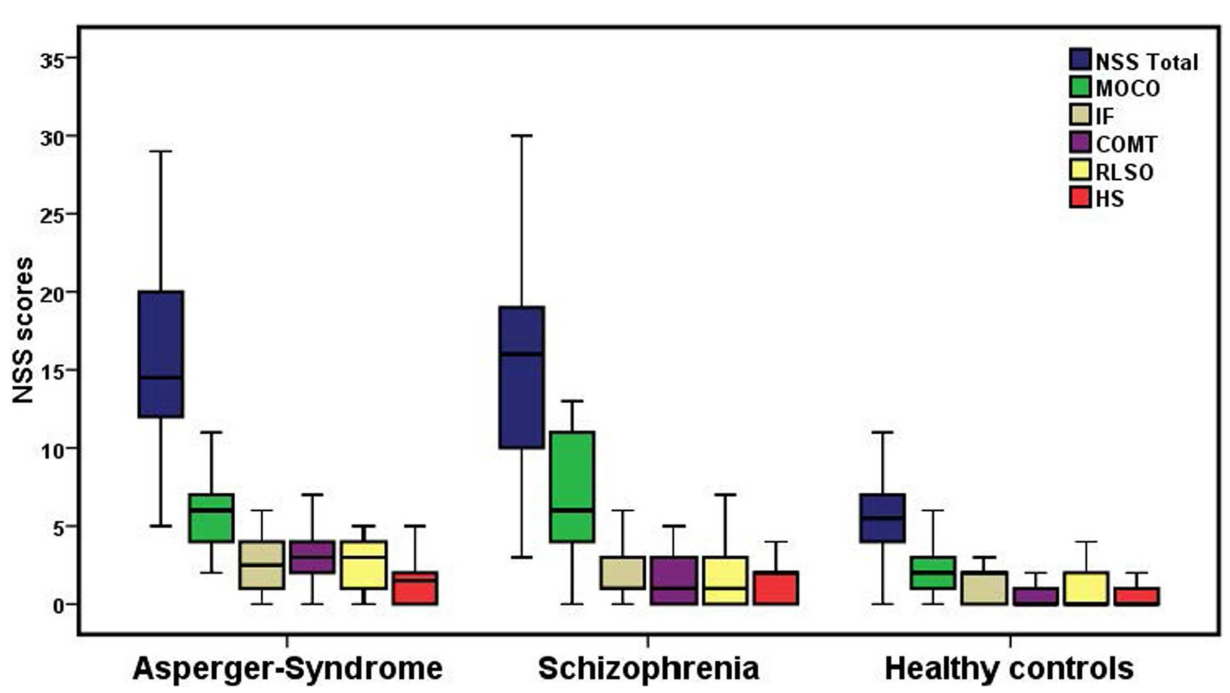

FIGURE 1 | Neurological soft signs total scores and NSS scores on the five subscales among the three groups. The bottom and top of the box represent the first and third quartile, and the band inside the box is the second quartile (the median). The ends of the whiskers indicate the minimum and maximum of the NSS performance. MOCO, motor coordination; COMT, complex motor tasks; IF, integrative functions; RLSO, right/left and spatial orientation; HS, hard signs. 
subscales IF $[F(4,47)=2.51 ; p=0.054]$, RLSO $[F(4,47)=1.5$; $p=0.217]$, and HS $[F(4,47)=1.1 ; p=0.364]$.

\section{AS VS. HC}

Compared with $\mathrm{HC}$, individuals with AS showed significantly higher NSS total scores $[F(3,48)=24.50 ; p<0.001]$ and elevated NSS on the subscales MOCO $[F(3,48)=15.29 ; p<0.001]$, IF $[F(3,48)=3.71 ; p=0.018], \operatorname{COMT}[F(3,48)=17.81 ; p<0.001]$, RLSO $[F(3,48)=6.21 ; p=0.001]$, and HS $[F(3,48)=3.25$; $p=0.03]$. NSS total and three subscale scores (MOCO, COMT, and RLSO) hold Bonferroni correction for multiple testing $(p<0.0041)$.

\section{SZ VS. HC}

Compared with HC, SZ patients showed significantly more total NSS signs $[F(4,47)=12.90 ; p<0.001]$ and higher NSS scores on the subscale MOCO $[F(4,47)=11.33 ; p<0.001]$, IF $[F(4$, $47)=2.59 ; p=0.048]$, and HS $[F(4,47)=4.48 ; p=0.004]$. NSS total and two subscale scores (MOCO and HS) hold Bonferroni correction for multiple testing $(p<0.0041)$. Additionally, no significant difference was found between SZ patients and $\mathrm{HC}$ on the subscale COMT $[F(4,47)=2.45 ; p=0.059]$ and RLSO $[F(4$, $47)=1.56 ; p=0.2]$.

\section{CLINICAL COMPARISONS}

In patients with SZ, SAPS, SANS, and BPRS scores were not significantly associated with total score and five subscores of NSS at the conventional significance level $(p<0.05)$. In individuals with AS, BPRS scores were not associated with total score and five subscores of NSS at the conventional significance level $(p<0.05)$.

\section{DISCRIMINANT ANALYSES OF NSS PERFORMANCE}

In this study, we conducted a LDA $(11,49,50)$, to examine the ability of the total NSS and five subscale scores to discriminate between the three groups. With this method, we also tested for the possibility of predicting the correct diagnosis solely based on NSS performance. Only the NSS subscales that reached statistical significance in post hoc analysis and survived the Bonferroni correction were used as predictive variables.

Using predictive LDA, we found that $71.8 \%$ of the cases were correctly classified in terms of the group as a function of the total NSS and two subscale scores (MOCO and COMT). The individual discriminant rates for each diagnostic group were $92.3 \%$ for HC, $69.2 \%$ for individuals with AS, and $53.8 \%$ for SZ patients. The results of the descriptive LDA revealed the emergence of two significant linear discriminant functions: function 1 (Wilks' $\lambda=0.495 ; \chi^{2}=52.106 ; p<0.001$; eigenvalue: 0.627 ; canonical correlation $=0.621$ ) could explain $72.1 \%$ of the variance, while function 2 (Wilks' $\lambda=0.805 ; \chi^{2}=16.084 ; p<0.001$; eigenvalue: 0.243 ; canonical correlation $=0.442$ ) could only explain $27.9 \%$ of the variance. The distribution of the three groups is shown in Figure 2 and function 1 and function 2 are illustrated.

In this study, predictive LDA was also conducted for groupgroup comparison. All three analyses showed significant results.

\section{AS VS. SZ}

One of them was between individuals with AS and SZ patients. In this analysis, only the NSS subscale MOCO that reached statistical

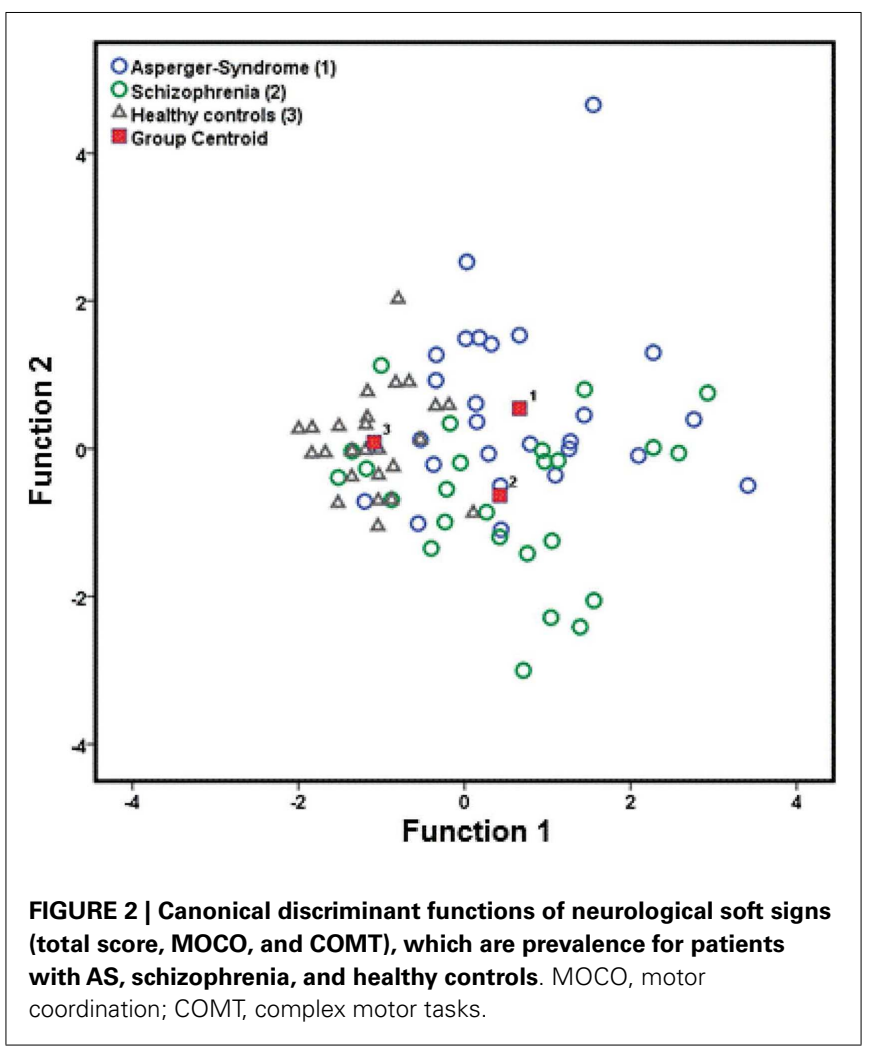

significance in the post hoc analysis and survived the Bonferroni correction was used as predictor variable. The total correct discriminant rate was $61.5 \%$. The results of the descriptive LDA revealed the emergence of non-significant linear discriminant function (Wilks' $\lambda=0.994 ; \chi^{2}=0.283 ; p=0.595$; eigenvalue: 0.006; canonical correlation $=0.076$ ). Because of the nonsignificant discriminant function between individuals with AS and patients with SZ, we re-ran the LDA by adding two more NSS variables, which did not survive the Bonferroni correction (11). After including the NSS total score and the NSS subscale COMT in the LDA, the correct discriminant rate elevated from 61.5 to $71.2 \%$ (Wilks' $\lambda=0.774 ; \chi^{2}=12.438 ; p=0.006$; eigenvalue: 0.292 ; canonical correlation $=0.476$ ). The correct rate of AS individuals was $65.4 \%$, while the correct rate of SZ patients was $76.9 \%$.

\section{AS VS. HC}

The other was between individuals with AS and HC. In this analysis, total NSS and three subscale scores (MOCO, COMT, and HS) that reached statistical significance in the post hoc analysis and survived the Bonferroni correction were used as predictor variables. The total correct discriminant rate was $92.3 \%$. The descriptive LDA revealed the emergence of one significant linear discriminant function (Wilks' $\lambda=0.445 ; \chi^{2}=37.746 ; p<0.001$; eigenvalue: 1.195 ; canonical correlation $=0.738)$.

\section{SZ VS. HC}

The remaining predictive LDA was between SZ patients and HC. In this analysis, total NSS and three subscale scores (MOCO and HS) that reached statistical significance in the post hoc analysis and survived the Bonferroni correction were used as predictor variables. 
The total correct discriminant rate was $80.8 \%$. The results of the descriptive LDA revealed the emergence of one significant linear discriminant function (Wilks' $\lambda=0.569 ; \chi^{2}=27.334 ; p<0.001$; eigenvalue: 0.757 ; canonical correlation $=0.656$ ).

\section{POTENTIAL INFLUENCE OF MEDICATION}

In patients with SZ, NSS total scores $(r=0.335 ; p=0.095)$ and scores on the subscales MOCO $(r=0.262 ; p=0.228)$, COMT $(r=0.133 ; p=0.547)$, HS $(r=0.255 ; p=0.240)$, IF $(r=0.289$; $p=0.182)$, and RLSO $(r=0.140 ; p=0.523)$ were not associated with $\mathrm{CPZ}$ equivalents at the conventional significance level $(p<0.05)$.

\section{DISCUSSION}

This study assessed and compared NSS levels in both patients with SZ and AS. Two main findings emerged: first, patients with SZ show significantly higher NSS score on the subscale MOCO when compared to individuals with AS. Second, SZ patients can be distinguished from those with AS by only one NSS subscale (MOCO). These findings were consistent across the analyses of prevalence and in the LDA.

Previous studies on motor abnormalities in AS clearly underestimated the prevalence of NSS in this syndrome and focused exclusively on rather complex movement disorders. In fact, only two previous studies investigated the severity of NSS in AS (33, 34). In the study conducted by Tani (33), individuals with AS had significantly higher NSS total and complex motor acts scores when compared to the control group. The authors concluded that NSS represent a non-specific vulnerability factor for AS (33). More recently, Mayoral (34) investigated 30 patients with early-onset SZ and 29 individuals with AS. In agreement with our results, they found that individuals with AS have higher NSS scores than HC. Second, however, the authors concluded that there are no significant differences between both patient groups in any of the NSS scores. However, it is possible that the discordant findings reported by Mayoral (34) were due to large differences in sociodemographic variables among the study participants. In fact, the IQ levels in the control group were significantly higher than in SZ patients and individuals with AS. Hence, some patients with AS were taking antipsychotic medication, a fact that may have biased particular NSS tasks in this group. Last but not least, recent research indicates that only $5 \%$ of SZ patients have a psychosis onset before age of 15 years (51). Therefore, investigating young patients with early-onset SZ does not allow making inferences for the whole SZ spectrum, because NSS might be instable in subgroups of young patients with incomplete brain maturation. Thus, the above mentioned findings cannot be generalized to the whole autism spectrum.

To some extent, our findings are consistent with the two above mentioned NSS studies. In line with results presented by Tani (33), we found that individuals with AS exhibit higher NSS levels on the subscale MOCO, COMT, and RLSO when compared to HC. However, we did not find any significant difference between NSS levels on the subscale HS. In contrast to Mayoral (34), who found no differences between SZ and patients with AS, we observed significantly higher NSS scores on the subscale MOCO in SZ when compared to individuals with AS. Compared with both previous studies, our findings are likely to be more robust because of three reasons: first, individuals with AS were free of psychotropic medication. Though all our SZ patients were medicated, the negative results in correlations between NSS and CPZ further reduces potential concerns that our findings might be confounded by antipsychotic drug treatment. Furthermore, the duration of exposure to second-generation antipsychotic medication in SZ patients was rather low. A second strength of our study is that individuals with SZ and AS had low prevalence of acute psychiatric symptoms and, in addition, did not differ in BPRS scores as measures of psychopathology. We believe this to be important, as SZ patients with more severe psychotic symptoms have been shown to score higher on the NSS scale in comparison with SZ patients without any negative or positive symptoms (3). In fact, recent studies showed that patients with negative symptoms are characterized by more severe neurological abnormalities including different sensory-motor functions (52). For instance, a number of reports have also noted that $\mathrm{SZ}$ patients with negative symptoms exhibit higher prevalence of spontaneous movements $(53,54)$ or NSS (55-59). In conclusion, there is an association between NSS and negative symptoms in SZ. However, our SZ sample scored rather low on SANS and SAPS. Third, SZ patients and individuals with AS were of similar educational level. Given the large body of evidence in individuals with AS and SZ suggesting a significant relationship between intelligence and movement (60), we used years of education as a covariate when analyzing differences in NSS scores. In contrast to both aforementioned studies, our study sample comprised mainly young adults in a clinically stable disease state and rather advanced brain maturation. However, for the interpretation of the present results, it is important to bear in mind that the human brain undergoes a highly dynamic development, which continues into adulthood (61). While the majority of longitudinal studies on brain growth in autism focused on children, the trend of brain development in adolescence, and adulthood remains unidentified (62). Our data might support the hypothesis of developmental deficits in AS during adolescence and adulthood.

Several lines of scientific evidence suggest that AS and SZ have both unique and similar sensory-motor features. In particular, there is a stimulating debate whether these disorders are related conditions or not (37). The findings of our present study provide support for both positions. SZ patients exhibited significantly higher NSS levels on the subscale MOCO when compared to individuals with AS. The NSS subscale MOCO comprises both, tasks which involve small muscles of the hand, and tasks which necessitate a tight link between one's own bodily movement and the spatial-temporal constraints. This finding is of particular interest given recent evidence of individuals with SZ showing poor levels of motor dexterity (63). As such, this action is based on visual perception and fine motor precision. Therefore, our first finding supports the hypothesis that patients with SZ exhibit serious problems when using sensory information to guide and time fine finger and hand movements. Moreover, there is some evidence that abnormalities of fine MOCO have a developmental origin and manifest even in a group of clinical at-risk mental state individuals $(64,65)$. In fact, research on NSS in ultra-high risk (UHR) conditions for developing mental illness might also provide important clues for the understanding of motor abnormalities in 
psychotic disorders. Nevertheless, to date, only few studies investigated NSS in UHR individuals $(66,67)$. Leask and colleagues (67) concluded that NSS might precede SZ, but are not caused by infectious illness in early childhood. In the pioneer longitudinal neuroimaging study on UHR individuals, Mittal and colleagues (68) suggested a significant relationship between NSS and longitudinal cerebellar-thalamic tract integrity. As such, NSS might provide insight into the role of cognitive dysmetria in the highrisk period. These results are supported by previous research on infant motor development that considered childhood neuromotor dysfunction as a risk factor for SZ spectrum disorders (64, 69). In summary, NSS might be considered as an intrinsic part of vulnerability to psychosis and should be discussed as markers of disordered neurodevelopment in SZ (70).

After Bonferroni correction, no significant differences were found between SZ and AS in total NSS and four subscales comprising rather gross motor skills such as stait and gait, tandem walking, finger-to-nose test or right/left orientation. There are several explanations for the particular deficit in gross motor skills in both disorders. In order to properly perform gross bodily actions, the interaction of motor cortex, basal ganglia, and thalamus is critical. Recently, altered gray matter volumes within the limbic basal ganglia loop system (e.g., left thalamus, putamen) were found to be common in both $\mathrm{SZ}$ and autism $(37,71)$. We believe that these findings lend support to the theory of a disrupted basal ganglia loop system in both disorders and suggest that SZ and AS share a number of neurobiological similarities. Hence, our results provide arguments against the theory that $\mathrm{SZ}$ and $\mathrm{AS}$ are diametrically opposite ends of a continuum (72).

The present study employed LDA in order to test for unique disease related patterns of NSS and to explore the degree of accuracy to which these patterns could be used to statistically discriminate between SZ and AS. Although the NSS subscale MOCO was found to be the most important predictor involved in discriminating, among the two clinical groups, it only accounted for an overall $61.5 \%$ correct classification. These results implicate that the level of abnormalities in perception-action coupling as described by MOCO may serve as a valuable predictor when trying to differentiate between patients with SZ and AS. However, it is noteworthy that by combining the NSS subscale MOCO with the total NSS and COMT subscale score the correct discriminant rate elevated to $71.2 \%$ and revealed significant discriminant function. Because of our modest sample size, some findings diminished more than it would have probably been the case with a larger study group. Although the LDA consisting NSS total scores was significant, NSS subscales tended to fall below our cut-off of 0.05 as the determinant of significance. Still, our observation that the subscale MOCO has a significant discriminant power between SZ patients and individuals with AS supports earlier assumptions that abnormalities of motor dexterity per se might be a major characteristic of SZ (73). According to our results, especially motor tests evaluating fine motor skills and manual dexterity might be helpful when classifying and differentiating patients with SZ and individuals with AS.

\section{LIMITATIONS}

We acknowledge several potential limitations of this study such as the possible differences in IQ levels among the study participants.
It is thus possible that we missed small between-group effects due to missing IQ scores in SZ patients and the control group. Apart from this, deficits in global measures of cognition such as intelligence are common in SZ patients $(74,75)$. On the other side, it is a well-established finding that IQ levels do not change over the course of illness and that lower IQ is a stable trait in patients suffering from SZ (76-78). Furthermore, variables such as education, occupation, and age can contribute significantly to IQ values (79). In other words, we believe the recent evidence to clearly suggest years of education as being an appropriate and stable indicator of global cognition in SZ. Second, the relatively small number of participants in each study group limits the power of the LDA. Third, statistical analysis of the three groups revealed a significant difference in gender. According to Cai (80) and colleagues, higher NSS levels were observed in 14- and 15-years-old boys when compared with girls of the same age. Since the differences in NSS performance declined with increasing age, the authors concluded that young boys might experience a delay of brain maturation when compared to girls of similar age, and hence, this might cause higher NSS scores in the male group (80). In our study, there were no differences for age distribution among the three groups and the majority of our study subjects were young adults with completed brain maturation. Further, there were no significant differences between male and female participants in NSS performance among the three groups. Therefore, uneven gender distribution across diagnostic groups might have not directly impact upon results of the statistical analysis in this study. Fourth, all SZ patients had been exposed to antipsychotic medication. In order to partial out a putative dose-dependent effect of second-generation antipsychotics on NSS performance, SZ patients' CPZ were considered as potential confounders in the present study. Although $\mathrm{CPZ}$ doses were included as covariates in the statistical analyses, we cannot completely rule out the possibility that antipsychotic medication might have influenced the NSS performance to some degree. Still, an influence of medication in our study is unlikely as every SZ patient was treated with atypical neuroleptics, the duration of treatment was relatively short, and none of the subjects showed serious medication side effects. Furthermore, none of the individuals with AS was treated with second- or first-generation antipsychotics. Correspondingly, second-generation antipsychotic treatment or medication side effects seem to have no effect on NSS performance in SZ (81). Fifth, healthy participants were not explicitly screened for AS by means of a standardized test. However, no signs of autistic traits were observed in healthy subjects during clinical and diagnostic (DSM-IV) interviewing. Last but not least, our study sample comprised patients suffering from different subtypes of SZ, a point that might complicate the interpretation of our results (82). However, subgroups were too small to test for this potential influence. Moreover, it is important to bear in mind that our findings are preliminary and that they need to be replicated in larger samples.

\section{CONCLUSION}

Sensory-motor abnormalities in SZ and AS might manifest as NSS. Since NSS are present in both, individuals with SZ and AS, they may represent a putative neuromotor marker across the traditional diagnostic categorization. Understanding the role of NSS could 
help to gain further insight into the neurobiological underpinnings of SZ and AS. To this end, future studies should ideally combine thorough clinical, neurological, and psychopathological assessments with multi-modal neuroimaging techniques in order to elucidate how the respective factors relate to each other.

\section{AUTHOR CONTRIBUTIONS}

Dusan Hirjak, Philipp Arthur Thomann, Sabine C. Koch, and Thomas Fuchs designed the study and were involved in the interpretation of the results. Dusan Hirjak, Robert Christian Wolf, Katharina Maria Kubera, and Tanja Traeger performed statistical analyses. Dusan Hirjak, Philipp Arthur Thomann, Laura Mehl, and Janna K. Kelbel undertook neurological, psychopathological and psychometric assessments. Dusan Hirjak, Philipp Arthur Thomann, Katharina Maria Kubera and Robert Christian Wolf wrote the manuscript. All authors contributed to and have approved the final manuscript.

\section{ACKNOWLEDGMENTS}

The authors cordially thank all patients and healthy controls for participating in this study. This work was supported by the MarieCurie Initial Training Network TESIS: "Toward an Embodied Science of InterSubjectivity” (FP7-PEOPLE-2010-ITN, 264828). We also acknowledge financial support by Deutsche Forschungsgemeinschaft (DFG) and University of Heidelberg within the funding program "Open Access Publishing”.

\section{REFERENCES}

1. Heinrichs DW, Buchanan RW. Significance and meaning of neurological signs in schizophrenia. Am J Psychiatry (1988) 145:11-8.

2. Schroder J, Niethammer R, Geider FJ, Reitz C, Binkert M, Jauss M, et al. Neurological soft signs in schizophrenia. Schizophr Res (1991) 6:25-30. doi:10.1016/ 0920-9964(91)90017-L

3. Jahn T, Hubmann W, Karr M, Mohr F, Schlenker R, Heidenreich T, et al. Motoric neurological soft signs and psychopathological symptoms in schizophrenic psychoses. Psychiatry Res (2006) 142:191-9. doi:10.1016/j.psychres.2002.12.003

4. Chan RC, Xu T, Heinrichs RW, Yu Y, Wang Y. Neurological soft signs in schizophrenia: a meta-analysis. Schizophr Bull (2010) 36:1089-104. doi:10.1093/ schbul/sbp011

5. Bombin I, Arango C, Buchanan RW. Significance and meaning of neurological signs in schizophrenia: two decades later. Schizophr Bull (2005) 31:962-77. doi:10.1093/schbul/sbi028

6. Bolton D, Gibb W, Lees A, Raven P, Gray JA, Chen E, et al. Neurological soft signs in obsessive compulsive disorder: standardised assessment and comparison with schizophrenia. Behav Neurol (1998) 11:197-204. doi:10.1155/1999/639045

7. Jaafari N, Baup N, Bourdel MC, Olie JP, Rotge JY, Wassouf I, et al. Neurological soft signs in OCD patients with early age at onset, versus patients with schizophrenia and healthy subjects. J Neuropsychiatry Clin Neurosci (2011) 23:409-16. doi:10.1176/appi.neuropsych.23.4.409

8. Mohr F, Hubmann W, Cohen R, Bender W, Haslacher C, Honicke S, et al. Neurological soft signs in schizophrenia: assessment and correlates. Eur Arch Psychiatry Clin Neurosci (1996) 246:240-8. doi:10.1007/BF02190275

9. Kinney DK, Yurgelun-Todd DA, Woods BT. Neurologic signs of cerebellar and cortical sensory dysfunction in schizophrenics and their relatives. Schizophr Res (1999) 35:99-104. doi:10.1016/S0920-9964(98)00121-2

10. Rigucci S, Dimitri-Valente G, Mandarelli G, Manfredi G, Comparelli A, Filippis SD, et al. Neurological soft signs discriminate schizophrenia from bipolar disorder. J Psychiatr Pract (2014) 20:147-53. doi:10.1097/01.pra.0000445250. 20875.0e

11. Zhao Q, Ma YT, Lui SS, Liu WH, Xu T, Yu X, et al. Neurological soft signs discriminate schizophrenia from major depression but not bipolar disorder. Prog Neuropsychopharmacol Biol Psychiatry (2013) 43:72-8. doi:10.1016/j.pnpbp.2012. 12.006
12. Krebs MO, Gut-Fayand A, Bourdel M, Dischamp J, Olie J. Validation and factorial structure of a standardized neurological examination assessing neurological soft signs in schizophrenia. Schizophr Res (2000) 45:245-60. doi:10.1016/S09209964(99)00206-6

13. Thomann PA, Wustenberg T, Santos VD, Bachmann S, Essig M, Schroder J. Neurological soft signs and brain morphology in first-episode schizophrenia. Psychol Med (2009) 39:371-9. doi:10.1017/S0033291708003656

14. Hirjak D, Wolf RC, Stieltjes B, Hauser T, Seidl U, Schroder J, et al. Cortical signature of neurological soft signs in recent onset schizophrenia. Brain Topogr (2014) 27:296-306. doi:10.1007/s10548-013-0292-z

15. Zhao Q, Li Z, Huang J, Yan C, Dazzan P, Pantelis C, et al. Neurological soft signs are not "soft" in brain structure and functional networks: evidence from ALE meta-analysis. Schizophr Bull (2014) 40:626-41. doi:10.1093/schbul/sbt063

16. Dazzan P, Morgan KD, Orr KG, Hutchinson G, Chitnis X, Suckling J, et al. The structural brain correlates of neurological soft signs in AESOP first-episode psychoses study. Brain (2004) 127:143-53. doi:10.1093/brain/awh015

17. Janssen J, Diaz-Caneja A, Reig S, Bombin I, Mayoral M, Parellada M, et al. Brain morphology and neurological soft signs in adolescents with first-episode psychosis. Br J Psychiatry (2009) 195:227-33. doi:10.1192/bjp.bp.108.052738

18. Thomann PA, Roebel M, Dos Santos V, Bachmann S, Essig M, Schroder J. Cerebellar substructures and neurological soft signs in first-episode schizophrenia. Psychiatry Res (2009) 173:83-7. doi:10.1016/j.pscychresns.2008.07.006

19. Hirjak D, Wolf RC, Stieltjes B, Seidl U, Schroder J, Thomann PA. Neurological soft signs and subcortical brain morphology in recent onset schizophrenia. J Psychiatr Res (2012) 46:533-9. doi:10.1016/j.jpsychires.2012.01.015

20. Hirjak D, Wolf RC, Stieltjes B, Hauser T, Seidl U, Thiemann U, et al. Neurological soft signs and brainstem morphology in first-episode schizophrenia. Neuropsychobiology (2013) 68:91-9. doi:10.1159/000350999

21. Bleuler E. Dementia Praecox or the Group of Schizophrenias. Zinkin J, Trans. New York, NY: International Universities Press (1950).

22. Parnas J. The core Gestalt of schizophrenia. World Psychiatry (2012) 11:67-9. doi:10.1016/j.wpsyc.2012.05.002

23. Parnas J. A disappearing heritage: the clinical core of schizophrenia. Schizophr Bull (2011) 37:1121-30. doi:10.1093/schbul/sbr081

24. Cappon D. Clinical manifestations of autism and schizophrenia in childhood. Can Med Assoc J (1953) 69:44-9.

25. Pelletier AL, Mittal VA. An autism dimension for schizophrenia in the next Diagnostic and Statistical Manual? Schizophr Res (2012) 137:269-70. doi:10.1016/j. schres.2012.01.033

26. Kanner L. Early infantile autism. J Pediatr (1944) 25:211-7. doi:10.1016/S00223476(44)80156-1

27. Asperger H. Die "Autistischen Psychopathen" im kindesalter. Arch Psychiatr Nervenkr (1944) 117:76-136. doi:10.1007/BF01837709

28. Barneveld PS, Pieterse J, De Sonneville L, Van Rijn S, Lahuis B, Van Engeland $\mathrm{H}$, et al. Overlap of autistic and schizotypal traits in adolescents with Autism Spectrum Disorders. Schizophr Res (2011) 126:231-6. doi:10.1016/j.schres.2010. 09.004

29. Konstantareas MM, Hewitt T. Autistic disorder and schizophrenia: diagnostic overlaps. J Autism Dev Disord (2001) 31:19-28. doi:10.1023/A:1005605528309

30. Bevan Jones R, Thapar A, Lewis G, Zammit S. The association between early autistic traits and psychotic experiences in adolescence. Schizophr Res (2012) 135:164-9. doi:10.1016/j.schres.2011.11.037

31. Waris P, Lindberg N, Kettunen K, Tani P. The relationship between Asperger's syndrome and schizophrenia in adolescence. Eur Child Adolesc Psychiatry (2013) 22:217-23. doi:10.1007/s00787-012-0338-x

32. Rinehart NJ, Tonge BJ, Bradshaw JL, Iansek R, Enticott PG, Mcginley J. Gait function in high-functioning autism and Asperger's disorder: evidence for basal-ganglia and cerebellar involvement? Eur Child Adolesc Psychiatry (2006) 15:256-64. doi:10.1007/s00787-006-0530-y

33. Tani P, Lindberg N, Appelberg B, Nieminen-Von Wendt T, Von Wendt L, PorkkaHeiskanen T. Clinical neurological abnormalities in young adults with Asperger syndrome. Psychiatry Clin Neurosci (2006) 60:253-5. doi:10.1111/j.1440- 1819. 2006.01494.x

34. Mayoral M, Merchan-Naranjo J, Rapado M, Leiva M, Moreno C, Giraldez M, et al. Neurological soft signs in juvenile patients with Asperger syndrome, earlyonset psychosis, and healthy controls. Early Interv Psychiatry (2010) 4:283-90. doi:10.1111/j.1751-7893.2010.00197.x

35. Whyatt C, Craig C. Sensory-motor problems in Autism. Front Integr Neurosci (2013) 7:51. doi:10.3389/fnint.2013.0005 
36. Esposito G, Pasca SP. Motor abnormalities as a putative endophenotype for Autism Spectrum Disorders. Front Integr Neurosci (2013) 7:43. doi:10.3389/ fnint.2013.00043

37. Cheung C, Yu K, Fung G, Leung M, Wong C, Li Q, et al. Autistic disorders and schizophrenia: related or remote? An anatomical likelihood estimation. PLoS One (2010) 5:e12233. doi:10.1371/journal.pone.0012233

38. Wittchen HU, Wunderlich U, Gruschwitz S, Zaudig M. SKID-I: Strukturiertes Klinisches Interview für DSM-IV. Göttingen: Hogrefe (1997).

39. Lord C, Rutter M, Goode S, Heemsbergen J, Jordan H, Mawhood L, et al. Autism diagnostic observation schedule: a standardized observation of communicative and social behavior. J Autism Dev Disord (1989) 19:185-212. doi:10.1007/BF02211841

40. Weiß RH, editor. CFT 20-R Grundintelligenztest Skala 2 - Revision. Göttingen: Hogrefe (2006).

41. Miller TJ, Mcglashan TH, Rosen JL, Cadenhead K, Cannon T, Ventura J, et al. Prodromal assessment with the structured interview for prodromal syndromes and the scale of prodromal symptoms: predictive validity, interrater reliability, and training to reliability. Schizophr Bull (2003) 29:703-15. doi:10.1093/oxfordjournals.schbul.a007040

42. Bachmann S, Bottmer C, Schroder J. Neurological soft signs in first-episode schizophrenia: a follow-up study. Am J Psychiatry (2005) 162:2337-43. doi:10. 1176/appi.ajp.162.12.2337

43. Oldfield RC. The assessment and analysis of handedness: the Edinburgh inventory. Neuropsychologia (1971) 9:97-113. doi:10.1016/0028-3932(71)90067-4

44. Overall JE, Gorham DR. The brief psychiatric rating scale. Psychol Rep (1962) 10:799-812. doi:10.2466/pr0.1962.10.3.799

45. Andreasen NC. The Scale for the Assessment of Positive Symptoms (SAPS). Iowa City: University of Iowa (1984).

46. Andreasen NC. The Scale for the Assessment of Negative Symptoms (SANS). Iowa City: University of Iowa (1983).

47. Strauss JS, Carpenter WT Jr. The prediction of outcome in schizophrenia. II. Relationships between predictor and outcome variables: a report from the WHO international pilot study of schizophrenia. Arch Gen Psychiatry (1974) 31:37-42. doi:10.1001/archpsyc.1974.01760130021003

48. Hall RC. Global assessment of functioning. A modified scale. Psychosomatics (1995) 36:267-75. doi:10.1016/S0033-3182(95)71656-5

49. Fisher RA. The use of multiple measurements in taxonomic problems. Ann Hum Genet (1936) 7:179-88.

50. Arango C, Bartko JJ, Gold JM, Buchanan RW. Prediction of neuropsychological performance by neurological signs in schizophrenia. Am J Psychiatry (1999) 156:1349-57.

51. Pakyurek M, Yarnal R, Carter C. Treatment of psychosis in children and adolescents: a review. Adolesc Med State Art Rev (2013) 24:420-32, ix.

52. Galderisi S, Maj M. Deficit schizophrenia: an overview of clinical, biological and treatment aspects. Eur Psychiatry (2009) 24:493-500. doi:10.1016/j.eurpsy. 2009.03.001

53. Chen EY, Lam LC, Chen RY, Nguyen DG. Negative symptoms, neurological signs and neuropsychological impairments in 204 Hong Kong Chinese patients with schizophrenia. Br J Psychiatry (1996) 168:227-33. doi:10.1192/bjp.168.2.227

54. Telfer S, Shivashankar S, Krishnadas R, Mccreadie RG, Kirkpatrick B. Tardive dyskinesia and deficit schizophrenia. Acta Psychiatr Scand (2011) 124:357-62. doi:10.1111/j.1600-0447.2011.01751.x

55. Buchanan RW, Kirkpatrick B, Heinrichs DW, Carpenter WT Jr. Clinical correlates of the deficit syndrome of schizophrenia. Am J Psychiatry (1990) 147:290-4.

56. Galderisi S, Maj M, Mucci A, Cassano GB, Invernizzi G, Rossi A, et al. Historical, psychopathological, neurological, and neuropsychological aspects of deficit schizophrenia: a multicenter study. Am J Psychiatry (2002) 159:983-90. doi:10.1176/appi.ajp.159.6.983

57. Peralta V, Moreno-Izco L, Sanchez-Torres A, Garcia De Jalon E, Campos MS, Cuesta MJ. Characterization of the deficit syndrome in drug-naive schizophrenia patients: the role of spontaneous movement disorders and neurological soft signs. Schizophr Bull (2014) 40:214-24. doi:10.1093/schbul/sbs152

58. Buchanan RW, Breier A, Kirkpatrick B, Elkashef A, Munson RC, Gellad F, et al. Structural abnormalities in deficit and nondeficit schizophrenia. Am J Psychiatry (1993) 150:59-65.

59. Arango C, Kirkpatrick B, Buchanan RW. Neurological signs and the heterogeneity of schizophrenia. Am J Psychiatry (2000) 157:560-5. doi:10.1176/appi.ajp. 157.4.560
60. Dziuk MA, Gidley Larson JC, Apostu A, Mahone EM, Denckla MB, Mostofsky SH. Dyspraxia in autism: association with motor, social, and communicative deficits. Dev Med Child Neurol (2007) 49:734-9. doi:10.1111/j.1469-8749.2007. 00734.x

61. Giedd JN, Blumenthal J, Jeffries NO, Castellanos FX, Liu H, Zijdenbos A, et al. Brain development during childhood and adolescence: a longitudinal MRI study. Nat Neurosci (1999) 2:861-3. doi:10.1038/13158

62. Hua X, Thompson PM, Leow AD, Madsen SK, Caplan R, Alger JR, et al. Brain growth rate abnormalities visualized in adolescents with autism. Hum Brain Mapp (2013) 34:425-36. doi:10.1002/hbm.21441

63. Sasayama D, Hori H, Teraishi T, Hattori K, Ota M, Matsuo J, et al. Benzodiazepines, benzodiazepine-like drugs, and typical antipsychotics impair manual dexterity in patients with schizophrenia. J Psychiatr Res (2014) 49:37-42. doi:10.1016/j.jpsychires.2013.10.019

64. Cannon M, Jones P, Huttunen MO, Tanskanen A, Huttunen T, Rabe-Hesketh S, et al. School performance in Finnish children and later development of schizophrenia: a population-based longitudinal study. Arch Gen Psychiatry (1999) 56:457-63. doi:10.1001/archpsyc.56.5.457

65. Tamagni C, Studerus E, Gschwandtner U, Aston J, Borgwardt S, RiecherRossler A. Are neurological soft signs pre-existing markers in individuals with an at-risk mental state for psychosis? Psychiatry Res (2013) 210:427-31. doi:10.1016/j.psychres.2013.06.016

66. McGorry PD, Mcfarlane C, Patton GC, Bell R, Hibbert ME, Jackson HJ, et al. The prevalence of prodromal features of schizophrenia in adolescence: a preliminary survey. Acta Psychiatr Scand (1995) 92:241-9. doi:10.1111/j.1600-0447. 1995.tb09577.x

67. Leask SJ, Done DJ, Crow TJ. Adult psychosis, common childhood infections and neurological soft signs in a national birth cohort. Br J Psychiatry (2002) 181:387-92. doi:10.1192/bjp.181.5.387

68. Mittal VA, Dean DJ, Bernard JA, Orr JM, Pelletier-Baldelli A, Carol EE, et al. Neurological soft signs predict abnormal cerebellar-thalamic tract development and negative symptoms in adolescents at high risk for psychosis: a longitudinal perspective. Schizophr Bull (2014). doi:10.1093/schbul/sbt199

69. Murray GK, Jones PB, Moilanen K, Veijola J, Miettunen J, Cannon TD, et al. Infant motor development and adult cognitive functions in schizophrenia. Schizophr Res (2006) 81:65-74. doi:10.1016/j.schres.2005.08.016

70. Dazzan P, Murray RM. Neurological soft signs in first-episode psychosis: a systematic review. Br J Psychiatry Suppl (2002) 43:s50-7. doi:10.1192/bjp.181. 43.s50

71. Hirjak D, Wolf RC, Wilder-Smith EP, Kubera KM, Thomann PA. Motor abnormalities and basal ganglia in schizophrenia: evidence from structural magnetic resonance imaging. Brain Topogr (2014). doi:10.1007/s10548-014-0377-3

72. Crespi B, Badcock C. Psychosis and autism as diametrical disorders of the social brain. Behav Brain Sci (2008) 31:241-261;discussion261-320. doi:10. 1017/S0140525X08004214

73. Midorikawa A, Hashimoto R, Noguchi H, Saitoh O, Kunugi H, Nakamura K. Impairment of motor dexterity in schizophrenia assessed by a novel finger movement test. Psychiatry Res (2008) 159:281-9. doi:10.1016/j.psychres.2007. 04.004

74. Keefe RS, Fenton WS. How should DSM-V criteria for schizophrenia include cognitive impairment? Schizophr Bull (2007) 33:912-20. doi:10.1093/schbul/ sbm046

75. Irani F, Kalkstein S, Moberg EA, Moberg PJ. Neuropsychological performance in older patients with schizophrenia: a meta-analysis of cross-sectional and longitudinal studies. Schizophr Bull (2011) 37:1318-26. doi:10.1093/schbul/sbq057

76. Mockler D, Riordan J, Sharma T. Memory and intellectual deficits do not decline with age in schizophrenia. Schizophr Res (1997) 26:1-7.

77. Hijman R, Hulshoff Pol HE, Sitskoorn MM, Kahn RS. Global intellectual impairment does not accelerate with age in patients with schizophrenia: a crosssectional analysis. Schizophr Bull (2003) 29:509-17. doi:10.1093/oxfordjournals. schbul.a007023

78. Hedman AM, Van Haren NE, Van Baal GC, Brans RG, Hijman R, Kahn RS, et al. Is there change in intelligence quotient in chronically ill schizophrenia patients? A longitudinal study in twins discordant for schizophrenia. Psychol Med (2012) 42:2535-41. doi:10.1017/S0033291712000694

79. Dragovic M, Waters FA, Jablensky A. Estimating premorbid intelligence in schizophrenia patients: demographically based approach. Aust NZJ Psychiatry (2008) 42:814-8. doi:10.1080/00048670802277214 
80. Cai L, Zhu X, Yi J, Bai M, Wang M, Wang Y, et al. Neurological soft signs and their relationship with measures of executive function in Chinese adolescents. J Dev Behav Pediatr (2013) 34:197-203. doi:10.1097/DBP.0b013e3182825c41

81. Gupta S, Andreasen NC, Arndt S, Flaum M, Schultz SK, Hubbard WC, et al. Neurological soft signs in neuroleptic-naive and neuroleptic-treated schizophrenic patients and in normal comparison subjects. Am J Psychiatry (1995) 152: 191-6.

82. Walther S, Horn H, Razavi N, Koschorke P, Muller TJ, Strik W. Quantitative motor activity differentiates schizophrenia subtypes. Neuropsychobiology (2009) 60:80-6. doi:10.1159/000236448

Conflict of Interest Statement: The authors declare that the research was conducted in the absence of any commercial or financial relationships that could be construed as a potential conflict of interest.
Received: 13 May 2014; paper pending published: 06 June 2014; accepted: 14 July 2014; published online: 06 August 2014.

Citation: Hirjak D, Wolf RC, Koch SC, Mehl L, Kelbel JK, Kubera KM, Traeger T, Fuchs $T$ and Thomann PA (2014) Neurological abnormalities in recent-onset schizophrenia and Asperger-syndrome. Front. Psychiatry 5:91. doi: 10.3389/fpsyt.2014.00091

This article was submitted to Schizophrenia, a section of the journal Frontiers in Psychiatry.

Copyright (C) 2014 Hirjak, Wolf, Koch, Mehl, Kelbel, Kubera, Traeger, Fuchs and Thomann. This is an open-access article distributed under the terms of the Creative Commons Attribution License (CC BY). The use, distribution or reproduction in other forums is permitted, provided the original author(s) or licensor are credited and that the original publication in this journal is cited, in accordance with accepted academic practice. No use, distribution or reproduction is permitted which does not comply with these terms. 